\title{
Violence on psychiatric in-patient units: what can be done?
}

\author{
Zerrin Atakan
}

There is evidence of an increase in violence on in-patient units (James et al, 1990), compared with earlier studies (Fottrell, 1980). This is also reflected in the number of articles published on violence. Rosenbaum (1991) reviewed the literature from 1935 to 1988 . Before 1968 no articles were published but by 1988,32 had appeared. A review of leading textbooks during the same period found little or no reference to violence until the early 1970 s. This finding does not mean that violence did not exist in mental hospitals previously, yet it may not have been as frequent or thought sufficiently serious to warrant research interest. An additional factor, especially in the pre-neuroleptic era, may have been the containment of patient violence by staff violence.

\section{Why has the interest in violence increased?}

A number of explanations can be considered. These are discussed below.

\section{'Psychosis wards'}

Changes introduced by the Department of Health have led to major changes in how mental health services operate, especially with the implementation of Community Care. With the closure of large mental hospitals there has been a significant loss of beds. Inpatient units, especially in inner city areas, have become 'psychosis wards', where the atmosphere can be charged, the disturbance level very high and patients' duration of stay often short, to make room for other more disturbed patients (MILMIS project group, 1995). Research indicates that the best predictors of assaultiveness are disorder related variables (Miller et al, 1993). Schizophrenia, mania, organic psychoses, substance abuse and personality disorders are especially associated with violent behaviour. It is not suprising that wards, with a high turnover, high percentage of detained patients, high bed-occupancy rate and too many disturbed psychotic patients are exposed to violence.

\section{Court Diversion Schemes and lack of provision for medium secure beds}

Following the Reed Report and the development of court diversion schemes, additional patients with complex needs are being identified from the penal system and offered psychiatric treatment. Although most of these patients need out-patient care (Exworthy \& Parrott, 1994), some are diverted to in-patient units, with $72 \%$ being admitted to open wards or intensive treatment units (Guite, personal communication). These patients may have already been exposed to violence or in some cases are being charged with crimes related to violence. Although most can be managed in open ward settings, a small but significant minority require treatment in more secure environments, where specialised care and rehabilitation can be delivered. The lack of intensive treatment units and the great difficulty in finding medium secure beds are now daily problems for inner city psychiatrists. These patients, frustrated and overstimulated in highly charged acute admission wards, are more likely to display violence.

\section{Substance abuse}

With the widespread increase of substance abuse, the 'double diagnosis' is a common occurrence in most psychiatric wards, especially in large cities. Some of the most vulnerable young patients, who have almost nothing else going for them, misuse various substances such as crack-cocaine, cannabis, amphetamines and alcohol. These alone can induce aggressive behaviour but their use also means that the violent culture of the drug scene' is brought to the ward. 
Ward milieu and staff related factors

The World Health Organization's Expert Committee on Mental Health concluded that the ward milieu is the single most important factor influencing the outcome of treatment. The ward milieu is created by its staff and patients and their attitudes, education, experience, cultural belief systems and, very importantly, the design of the facility itself. The literature on violence in mental hospitals suggests that the social environment of the ward, the organisation of activities and the behaviour of staff all affect the presence or absence of ward violence. Anxiety, negative attitudes, poor communication and conflict among staff can lead to aggressive behaviour among patients. Violence is more common and extreme in wards where staff functions are unclear and events such as activities, meetings or staff-patient encounters are unpredictable (Katz \& Kirkland, 1990). In the same study violence was found to be less common and less extreme in wards characterised by strong psychiatric leadership, clearly structured staff roles and a predictable environment.

Provision of well trained staff and adequate staff: patient ratios are also thought to be important elements in the prevention of violence. The disturbed patient, who may be frightened of his own aggressive impulses, requires a stable, consistent and confident environment, where help and support is available. James et al (1990) showed a $240 \%$ increase in violence in their 'high-dependency' unit over a 15 month period, which was strongly associated with an increase in the use of temporary nursing staff.

\section{The media and Supervision Registers}

Over recent years there has been a major change in the coverage of psychiatric patients violence in the media, arising perhaps from public anxiety about Community Care policies. The introduction of Supervision Registers, following the Clunis Report, has brought with it an increased focus on violence. There is an expectation that violence can be predicted and prevented, and that psychiatrists are responsible for doing so.

\section{Social factors}

Violence has a greater place in our daily lives. Tragic human attrocities now appear regularly on television. We witness the 'baddies win' and watch films in which violence is apparently justified and often glorified. This must affect our acceptance of its ongoing existence. Society's attitude towards violence is an important factor and one can speculate that this is being carried over into psychiatric wards.

\section{How can we prevent and manage violence in psychiatric wards?}

If there is increasing violence on psychiatric wards, then our primary task should be to look at ways of reducing it. Developing skills in this area would also help us to identify and prevent violence committed by some patients in the community following their discharge. Some strategies are suggested below.

\section{Provision of more Intensive Care or High Dependency Units}

Clinicians and managers should actively seek provision of units where highly disturbed patients can be treated by adequate numbers of specifically trained and committed staff. Control and restraint techniques should be well assimilated by all staff and refresher courses given on a regular basis. The design of these units should ensure that all safety measures are incorporated and that the patient has his own personal space. Sufficient provision of these units would also ease the pressure and stress levels currently experienced on open wards, where violence can be contagious, spreading to non-violent patients.

\section{Identification and measurement of violence}

The first task on existing wards should be to identify the extent and nature of the problem. At present most completed violent incident forms serve an administrative rather than a clinical purpose. The application of locally developed, easy to use measurements would be extremely useful. These may be based on reliable, established schedules such as the Overt Aggression Scale (Yudofsky et al, 1986), the Staff Observation Aggression Scale (Palmstierna \& Wistedt, 198) or the Scale for the Assessment of Aggressive and Agitated Behaviours (Brizer et al, 1987). Recording should be carried out on a regular basis in order to identify trends and emerging problems. 
Sexual harrassment and violence on wards is another serious concern, where underreporting is common. These incidents should be recorded on a routine basis. As well as having a policy on 'relationships', wards should also have clear guidelines on action to be taken when sexual violations do occur.

\section{Developing clear policies, ward structures and activities}

The literature shows that disturbed patients with a chaotic inner psyche can respond better to a structured, calm, predictable and confident milieu, where they feel protected and are occupied with enriching activities. Staff should have a clear understanding of models of violence theories, with specific attention to causes, preceding events, features and effects. These may be different for different patients. A clear policy in managing violence is an essential element, where each staff member knows exactly what needs to be done when there is a threat. There is enough evidence that patients are more likely to display aggression when they are bored and that fewer incidents are witnessed if they engage in occupational and industrial activities.

\section{Developing clear treatment models}

The potentially violent patient is often a young person, perhaps under the influence of drugs or alcohol, and is very likely to have a traumatic childhood history and disrupted education. In addition, he may be suffering from a severe mental disorder. A low selfesteem is common and due to previously experienced adversities he may be very sensitive to criticisms. Talking in a calm, non-threatening manner, where possible engaging the patient in taking some responsibility for his own treatment and allowing him to see that staff will help him control his own aggressive impulses, will help to reveal the individual buried under the layers of adversity.

It is obviously essential to treat the primary disorder, as this will alleviate the symptoms which may be causing the violent impulses. Potentially violent patients should not be made to feel all control is taken out of their hands, except in dangerously threatening situations. Putting patients into 'chemical straight-jackets', by administering very high doses of medication, is not a solution to staffing problems and may backfire with further displays of violence. Side effects should be carefully monitored, especially as signs such as akathisia are thought to be associated with violence (Crowner et al, 1990). A further reduction in arousal may be achieved using non-neuroleptic medication, such as benzodiazepines, lithium, propranolol or carbamazepine (Eichelman, 1988).

\section{Ensuring support and good communica- tion between staff members}

Any staff member working in a busy, emotionally charged psychiatric ward is under considerable pressure. Recruitment of both specially trained and committed nursing and medical staff is becoming a serious problem, especially in inner city areas. For this reason it is very important that a system of supervision and support groups is in place, where personal experiences of violence are discussed and shared on a regular basis. According to the literature, violence on wards is mostly directed towards nursing staff and this may lead to tension, alienation and poor communication between nursing and medical staff. In wards where there is strong leadership by the psychiatrist, staff feel supported and encouraged to develop new therapeutic skills and violence is generally less frequent and serious (Katz \& Kirkland, 1990).

\section{Conclusions}

Violence on wards is increasing. Some inner city wards have become 'untherapeutic' environments, where both staff and patients feel unsafe.

Strategies to reduce the level of violence include detailed recording of incidents, clear guidelines, structured programmes and ward activities, staff training and support. Team work and the commitment and leadership of the psychiatrist are essential.

Sufficient, well integrated in-patient, intensive care and medium secure units for the management of violent patients are essential counterparts of Community Care.

\section{Acknowledgements}

I am grateful to Tim Foster, Dr. Paul Wolfson and Dr. Rosalind Ramsay for their editorial assistance. 


\section{References}

Brizer, D. A., Convit, A., KRAKowsk, M., et al (1987) A rating scale for reporting violence on psychiatric wards. Hospital and Community Psychiatry, 38, 769-770.

Crowner, M. L., DouYon, R., Convr, A., et al (1990) Akathisia and violence. Psychopharmacology Bulletin. 26. 115-117.

EICHELMAN, B. (1988) Toward a rational pharmacotherapy for aggrestve and violent behavior. Hospital and Community Psychiatry. 39, 31-39.

EXWORTHY, T. \& PARROTT, J. (1994) Evaluation of a diversion from custody scheme at a Magistrate Court. Journal of Forensic Psychiatry, 4, 497-505.

FotTrELL, E. (1980) A study of violent behaviour among patients in psychiatric hospitals. British Joumal of Psychiatry, 136, 216-221.

James, D. G.. Fineberg, N. A., Shah, A. K., et al (1990) An increase in violence on an acute psychiatric ward, a study of associated factors. British Journal of Psychiatry. 166. 846-852.

KATZ. P. \& KIRKLAND. F. R. (1990) Violence and social structure on mental hospital wards. Psychiatry. 65 262-277.
Miluer, R. J., Zadolinny, K. \& Hafner, R. J. (1993) Profiles and predictors of assaultiveness for different psychiatric ward populations. American Journal of Psychiatry. 160. 1368-1373.

MILMIS PROJECT GrouP (1995) Monitoring inner London mental illness services. Psychiatric Bulletin. 19. 276-280.

PALMSTIERNA. T. \& WISTEDT, B. (1987) Staff observation aggression scale. SOAS: Prsentation and evaluation. Acta Psychiatrica Scandinavica, 76, 657-663.

RosenBAUM, M. (1991) Violence in psychiatric wards, role of the lax milieu. General Hospital Psychiatry. 13, 115-121.

YUDOFSKY, S. C., SILVER, J. M., JACKSON. W., et al (1986) The overt aggression scale for the objective rating of verbal and physical aggession. American Journal of Psychiatry. 143, 35-39.

Zerrin Atakan, Honorary Senior Lecturer and Consultant Psychiatrist, West Lambeth Community Care Trust, South Western Hospital, Pulross Road, Stockwell, London SW9 8EA

\section{Psychiatric Associations of the World}

Produced by the College Library, this updated register now includes details of over eighty psychiatric associations worldwide.

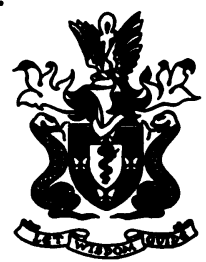

Psychiatric Associations of the World, 3rd Edition, Occasional Paper 29

Price $£ 5.00$

Available from the Publications Department, Royal College of Psychiatrists, 17 Belgrave Square, London SW1X 8PG (Tel. 0171-235 2351) 